\title{
Magnetic Nondestructive Testing Techniques of Constructional Steel
}

\author{
Er-gang Xiong ${ }^{a}$, Na-na Zhao, Zhao-qi Yan, Lan-wei Yang, Han He \\ School of Civil Engineering, Chang'an University, Xi'an 710061, P.R. China
}

\begin{abstract}
Steel is a kind of ferromagnetic material, which is extensively applied in such fields as buildings, bridges, railways, machines and lifeline engineering etc. Those engineering structures built of constructional steel will unavoidably experience some damages during their service lifetime, thus which will influence the distribution regularity of internal forces in structures, result in over-stresses, cause the local failure of structures, and even lead to collapse of the whole structure. Therefore, it is a pressing topic to study how to directly evaluate the real-time stressed states of structural members, damages and steel characteristics in present structural health monitoring and diagnosing fields. And the achievements of this research will be of theoretical significance and of application value of engineering. This paper summarizes varieties of new magnetic nondestructive testing techniques used in constructional steel, respectively investigates the testing principles, characteristics and application for the magnetic Barkhausen noise technique, magnetic acoustic emission technique, magnetic flux leakage technique, magnetic memory technique and magnetic absorption technique, and points out the problems present in the application of these new techniques to actual testing and the further research objective.
\end{abstract}

\section{Introduction}

With the continuous progress of science and technology and high-speed development of the social economy, the research on long-span space steel structures and steel bridges and its application have become more and more extensive. According to the related statistics, tens of large-scale gyms, hangars and exhibition halls will be constructed in recent years. The building areas of steel structures built every year have exceeded eight million M2. Furthermore, with the speedy advancement of economy, the demand for the long-span steel structures will be undoubtedly increasing. However, because such kind of structure is very sensitive to defects, has complicated load bearing and detailing characteristics, and it will be subjected to design load, construction quality, and environmental and man-made factors in their lifetime, the material property of structures will change(such as the welding thermal influence, corrosion, and initial defects etc.), and will unavoidably develop damages, which will deteriorate its health states, even lead to excessive internal forces and deformation thus to threaten the serviceability and safety of the whole structure. As a result, to ensure the serviceability and safety of the abovementioned structures and to satisfy the multi-performance requirements of people, it is necessary to conduct an in-line real-time monitoring and testing on the health states (including stresses, damages, and material property variation) of existing structures, so that the potential and newly-developed defects and damages can be observed as soon as possible in order to take corresponding measures of indispensable prevention and rehabilitation. Otherwise, disastrous results may occur. For instance, some tall building in Hongkong suddenly collapsed due to the change of stresses in each load bearing columns. Several bridges in Korea collapsed duet to overstresses. The roof of a gym in Hartford, Connecticut of USA also suddenly collapsed on a heavy snow night. The Jijiang Bridge of Chongqing in China suddenly collapsed due to quality. The roof with a 14.4-meter span of some heavy machine factory collapsed suddenly after two years operation in Liaoning province; in this accident, 42 people died, slight and serious wounded were up to 179 . Likewise, the catastrophic collapse accrued to 2E airport lounge of Charles de Gaulle in France due to quality of arch ceiling. The above accidents all caused heavy economic loss and social effect as illustrated in the work by authors (Du, 2004; Jonathan, 2005).

As a result, for long-span spaced steel structures and steel bridges, it is become an urgent research topic to realize in-line nondestructive testing or monitoring on the construction quality, damage, change of material property and actual stressed state.

\section{The research and application of magnetic nondestructive testing techniques}

The latest research results of modern microscopic electromagnetic mechanics indicates that the ferromagnetic material (i.e. constructional steel) will

\footnotetext{
${ }^{a}$ Corresponding author: xerg@chd.edu.cn
} 
exhibit different magnetic properties(magnetic flux, permeability, coercivity, magnetic induction intensity, magnetic memory effect and magnetic acoustic effect etc.) with different material properties, at different temperatures or in different stressed states such as austenite belongs to a paramagnetic phase, and martensite belongs to a ferromagnetic phase etc. Thus, the use of different types and intensities of magnetic field can contribute to the phase transformation of ferromagnetic materials. Furthermore, during the treating process, according to the requirements for material property, the metal crystal grain can also be refined to change their microstructure, such as the promotion of crystal reorganization of material, atom motion, slip, twinning etc. sequentially to influence or change the magnetic domain, domain wall, and magnetic domain structure of materials in order to control or improve the mechanical property of material. Contrariwise, when steel is constructed or used, the variation of their material properties or stressed states will inevitably induce the alteration of inner microscopic organization structure, thus to induce the variation of magnetic property of steel. Therefore, by the use of above magnetic effect of steel, the general regularity of its realtime distribution of internal force versus the change in magnetic property in order that the realtime nondestructive testing of a long-span steel structure and steel bridge can be performed.

As for the theory and application of magnetic nondestructive testing for ferromagnetic material, many experts at home and abroad emphasize this topic, have launched a lot of research work, and have obtained some achievements. However, most of their achievements are concentrated on aviation, machinery, petrol etc., and their research emphasis is centered on the damages detection of ferromagnetic materials like previous work by the authors (Maugin and Sabir, 1990; Gilanyi et al., 1998; Dong et al., 2006), the related theories and methods are as following.

\subsection{Theory and method of magnetic barkhausen noise}

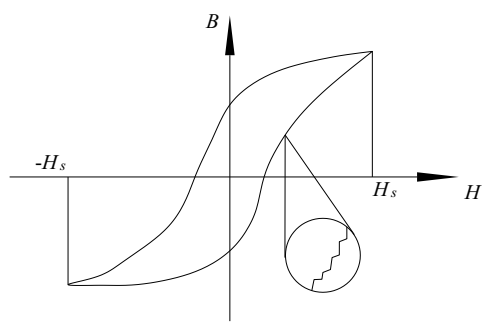

Figure 1. Barkhausen jump in the magnetic hysteretic curve

In 1919, Barkhausen found that the magnetization of ferromagnetic material presented a stepped type at the steepest region of magnetic hysteretic curve when it was magnetized, as shown in Figure 1. This stepped type of magnetization is an irreversible jump. This discontinuous magnetization stems from the discontinuous movement of magnetic domain and domain wall, which can generate a noisy signal in the detection coil called magnetic
Barkhausen noise (shortly named MBN). $\mathrm{MBN}$ is comparatively sensitive to the distribution of stresses and residual stresses. Like steel with a positive magnetostrictive coefficient, within the elastic region the signal of $\mathrm{MBN}$ will increase with the tensile stress and decrease with the compressive stress. The difference of loading signal and unloading signal can be used to measure the residual stress as shown in the work by $\mathrm{Mu}$ (1989). The earliest researches on the Barkhausen effect were all concerned with physical process, whose aim was to describe the movement of magnetic domain, and its measurement method was relatively simple. After 1960, because of the rapid development of electronic measurement techniques, many researchers have paid more profound investigation to the Barkhausen effect, and exploited quite a few fields.

In 1969, on the basis of Barkhausen effect, R.L. Pasley et al. studied the application of MBN to test stress distributions as shown in the work by Ju et al.(2003). In 1978, O. Sundstrom et al. investigated Barkhausen effect-based nondestructive testing of microscopic structural parameters, grain degree, iron loss, and stresses etc., as shown in the work by Park et al.(2000). In 1982, a new progress accrued to the MBN testing technique, and G.H. Kings et al. studied the grain orientation in stresses and noise spectrum emitted in each magnetization region by the $\mathrm{Si}$ steel as shown in the work by Krause et al.(1996). In that experiment, there were two methods: one was intensity spectrum of noise output, namely time domain analysis; the other was based on the amplitude domain technique, measuring the pulse distribution and productivity. Because the pulse output is similar to the Gause distribution, thus the statistical standard deviation of amplitude can be used to quantitatively study the stress response in different magnetization regions.

In recent years, although some researchers have utilized the MBN effect to test the grain degree, hardness, microstructure, stress, fatigue lifetime, defect and damage etc., as shown in the work by Dhar et al.(2001). The application of MBN to stress testing is primarily focused on the qualitative testing of stresses and residual stresses, but cannot permit an in-line realtime stress testing for ferromagnetic members. It is mainly because the magnitude of MBN signal will be influenced by the stress field at tested region, plastic deformation, grain orientation and varieties of inhomogeneous factors, which enables the relationship between MBN signal and stress not to be calibrated as shown in the work by Jagadish et al.(1990). Additionally the limited power spectrum of MBN will confine its testing depth suggested in previous work by the authors (Jiles, 1996; Lindgren and Lepisto, 2001).

\subsection{The theory and method of magnetic acoustic emission testing}

In the magnetization process of Ferromagnetic materials, the irreversible movement of the domain excited series of elastic wave pulses, in addition to producing the Barkhausen effect. This elastic wave pulse is analogous to mechanical acoustic emission, called magnetic acoustic 
emission (MAE) as shown in the work by Zhu (1991). MAE can be tested by a basic system shown in Figure 2 . The correlated researches demonstrate that MAE signal intensity is proportional to the volume, which produces inelastic strains. When the local external magnetic field is kept constant, MAE signal intensity varies from bearing stress, no matter how the stress is brought about by externally applied load or its residual stress. By the utilization of this property, we can scale MAE signal intensity of members in the steel structure without stresses when loading, and accordingly can make a nondestructive testing of a stress for the actual steel members in service as shown in the work by $\mathrm{Li}$ and Chen (2002).

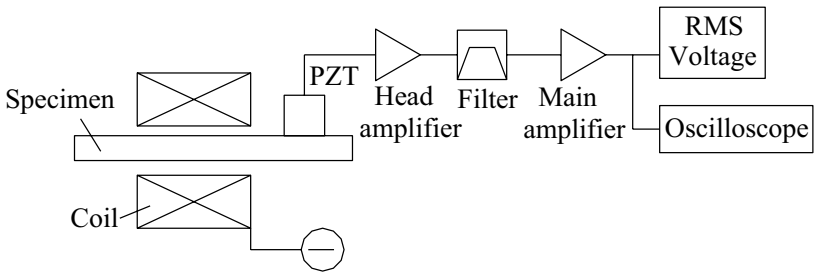

Figure 2. Diagram of the MAE testing system

MAE was found by Lord in 1975, who found that the movement of the domain wall in a Ni bar could produce an ultrasonic wave emission, when a $\mathrm{Ni}$ bar was under the action of DC (direct current) magnetic field as shown in the work by Hou et al.(1998). Later Higgin, Carpenter, Kusanagj et al. performed a lot of experiments successively, which further confirmed that MAE signal intensity of materials with stresses was lower than that of materials without stresses as shown in the work by Wang et al.(2005). Around in 1980, based on this, Kanji, Ono, Shibata et al. of the Canifornia Los Angels University in U.S.A launched a systematic research. They obtained different microscopic structures by using kinds of steel with different compositions, which were treated through different hot crafts. Under the action of different stresses, various kinds of parameters of MAE signal can be measured. It could be found that these parameters would be influenced by above factors. Some factors may even cause considerable influence. They scaled, compared the concrete materials, and then measured the residual stress of rails, which could be adopted as the basis of quality evaluation and had been put into the industrial application as shown in the work by Ma et al.(1996).

For MAE technique used to test the actual stresses of members, owing to a good many influencing factors, and complicated situations, this technique cannot be regarded as a universal testing method yet, which needs further studying in previous work by the authors (Anglada et al., 2001; Stewart et al., 2004).

\subsection{Theory and method of magnetic flux leakage}

In the magnetization process of Ferromagnetic materials, the presence of the defect will lead to the variation in the permeability, that is, the increasing magnetoresistance, which will make the magnetic flux in the magnetic circuit distort. One part of the flux will pass through the defect or pass around the defect inside, and the other part will leave the surface of the material and form a magnetic leakage field. By the direct use of the magnetometric devices, the magnetic leakage flux can be probed and recorded to realize a testing, which is called a magnetic leakage flux (MFL) technique studied in previous work by the authors (Xie and Peng, 2001; Wang et al., 2007). The diagram of testing principle with MFL is shown in Figure 3.

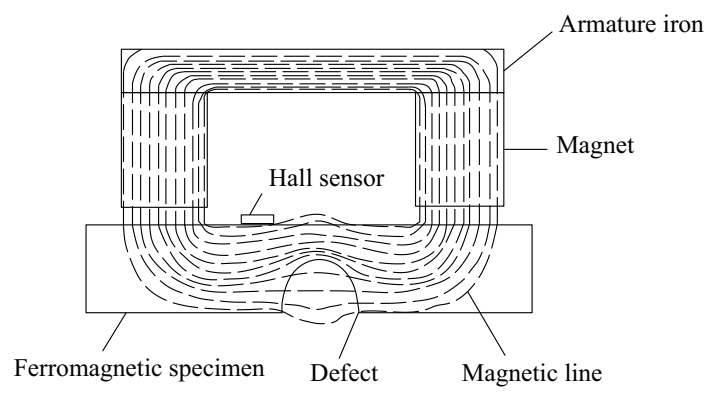

Figure 3. Diagram of the MFL testing principle

The foreign researches on MFL could be traced very early. Early in 1933, Zuschlug firstly addressed the application of magnetic sensitive sensor to measure MFL as shown in the work by Wang (2004), but not until 1947 did Hasting design the first MFL system, and until then the MFL testing was extensively recognized as shown in the work by Liu (2002). In 1950s, the manufactured MFL damage detection devices were invented by Foster in Germany as shown in the work by Plotnikov and Clapham (2002). In 1965, American TubecopeVetco International Corporation adopted the MFL detection device-Linalog, began a first damage testing on tubes and exploited a well detection system (wellcheck), by which the corrosion pits in the inner and outer diameter could be reliably detected, as well as transverse flaws and other types of defects as shown in the work by Jin et al. (2001). In 1973, the British Natural Gas Corporation utilized MFL to perform an in-service testing on the wall thickness thinning of the tube caused by corrosion for a natural gas pipe with $600 \mathrm{~mm}$. In that testing, the qualitative analysis was initially introduced. By the use of EMI MFL detection system made by ICO Corporation, combined with ultrasonic testing, the wall thickness, longitudinal and transverse defects etc. were desirably tested by the EMI MFL damage detection system, and thus a set of complete on-site magnetic damage detection technique was put forward as shown in the work by Foster (1982). Besides, Canadian Energy Technique Center utilized the MFL testing principle, managed to exploit a magnetic damage detecting device for steel wire rope, and realized a quantitative testing on the cross sectional loss, local defects of steel wire rope etc. Huazhong University of Science and Technology also successfully exploited a MFL detection device for the damages of steel wire rope as shown in the work by $\mathrm{Li}$ (2004)

In the MFL testing method, the MFL can usually be detected by magnetic particle or magnetic sensitive 
elements. The MFL detected by the magnetic particle is called a Magnetic particle inspection(MPI). The principle of MPI is that the dubious region of members are firstly polished and then excited by the magnetic field, through the observation of magnetic lines array, whether defects are present or not can be judged. This method is economical, reliable easily operated and is convenient for the defect judgment. At present it is still one of the main testing methods in our many industries. But the method also has several disadvantages, such as that it can only detect the surface defects of members, when magnetized by $\mathrm{DC}$, its testing thickness merely amounts to $1.5 \mathrm{~mm}$ etc., as shown in the work by Babbar et al. (2004).

By the use of magnetic sensitive elements to detect MFL, the magnitude of MFL at the defects can be obtained in order to possibly quantify defects and realize an intelligent recognition. Furthermore, the testing efficiency can be substantially increased, which can be utilized to perform a fast, overall testing of damages in previous work by the authors (Gwan and Eun, 2002; Philip et al., 2000; Thomas and Silvio, 2006).

\subsection{Theory and method of magnetic memory testing (MMT)}

The MMT method is a brand new diagnosis testing technique for ferromagnetic metal materials, which was put forward by Russian scholars represented by Dubov initiatively late in the 1920s, as shown in the work by Ren et al. (2000). According to the ferromagnetic theory, under the action of earth magnetic field, the magnetic conductivity at the defects of members will reduce, and the leakage magnetic filed of members' surfaces will increase, this characteristics of ferromagnetic materials is called magnetomechanical effect. The existence of the magnetomechanical effect can make the magnetic field strengthen, and simultaneously this strengthened magnetic field can memorize the locations of defects or stress concentrations of members, which is the so-called magnetic memory effect in previous work by the authors (Ren et al., 2000; Li and Huang, 2003). Because of the magnetomechanical effect present in ferromagnetic members, thus the distribution of the magnetic field of its surface is correspondingly related to stress loads applied on the members. So the defects and stress concentrations in the members can be indirectly diagnosed by testing the magnetic field distribution of members' surfaces, which is the basic principle of MMT, as shown in Figure 4. MMT technique is the only feasible NDT method which can diagnose metal members in the early phase till now. It has following main features: 1) not only macrodefects, but microdefects can be tested, and future danger can be predicted by this method, which is the most advantage of this technique; 2) it can perform a reliable testing on ferromagnetic members without specialized magnetization devices; 3) it requires no disposal, polishing, and other predisposal on the surfaces of members tested; 4) lift-off effect has a little influence on MMT, and gap variation arranging from a few millimeters to tens of millimeters will not affect testing results so much; 5) the facility is light, easy to operate, of high sensitivity, and of good repeatability and reliability as shown in the work by Ren et al.(2000).

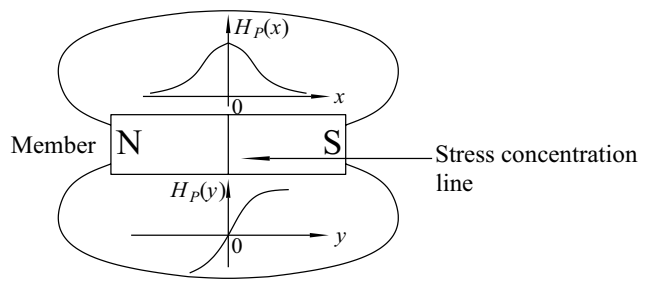

Figure 4. Diagram of the MMT principle

Until now some experts and scholars have applied the magnetic memory method(MMM) to detect the damages of pipelines, turbulent equipments as shown in previous work by the authors (Dubov, 2001; Dubov et al., 2003), and studied the magnetomechanical behavior with MMM as shown in the work by Dubov and Demin (1999), and simultaneously investigated the metal magnetic memory effect used to diagnose the metal property as shown in the work by Dubov (1997), yielded the basic parameters required to test the member by MMM as shown in the work by Dubov and Vstovskyy (1999), studied the characteristic of diffusion field when the defected members were subjected to forces as shown in the work by Dubov and Kouleev (2001). Through the research, they give the method to determine the residual stress of ferromagnetic members as shown in the work by Zhang et al. (2006), studied how to determine the stress-strain state by the use of diffusion field as shown in the work by Dubov (2000), performed a research of MMT on fatigue damages as shown in the work by Xing et al. (2006), and gave the determination method of limit state at the stress concentration region as well as shown in the work by Dubov (2002); besides, they studied the application of MMM to control welding quality in previous work by the authors (Dubov, 2002; Dubov, 2005).

The MMT technique is the only feasible NDT method which can diagnose metal members in the early phase till now, no matter whether for macrodefects or for those latent discontinuities which haven't taken shape, MMT can be an effective method. However, MMT can only permit the detection of defects and damages and cannot permit an in-line realtime stress testing on the ferromagnetic members.

\subsection{Theory and method of magnetic absorption testing}

The magnetic absorption testing theory and method means that: to place a ferromagnetic member in a coil field, then to measure the energy variation absorbed from radio-frequency coil field and finally quantitatively test the change of stress. However, this energy variation is associated with the biased magnetic field applied to the member in previous work by the authors (Hou et al., 1998; William, 1989). Its testing principle diagram is shown in Figure 5. 


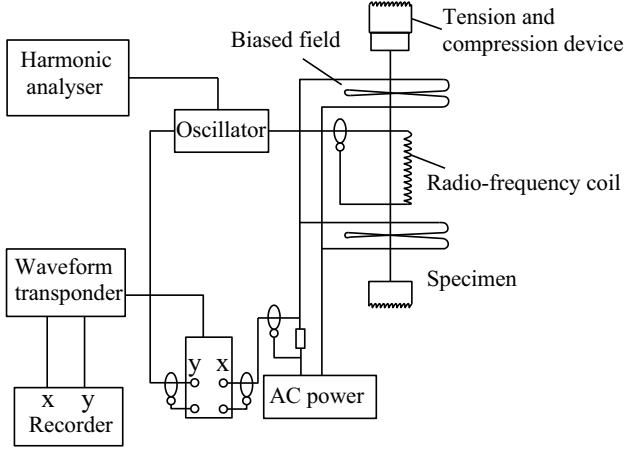

Figure 5. Diagram of magnetic absorption testing principle

The stress will change the orientation of magnetic domain when a tensile force is loaded to a saturated material with a positive magnetostrictive coefficient, some parallel magnetic domains will rotate their direction to be parallel or antiparallel to the applied field; on the other hand, when a tensile force is applied to a satuarated material with a negative magnetostrictive coefficient, some parallel magnetic domains will rotate a 900 angle to be perpendicular to the applied field. Thus, the signal-peak-peak amplitude and shape of magnetic absorption will depend on the direction of biased field to applied force and the sign of magnetostriction ferromagnetic materials possess.

The researches on various kinds of materials have indicated that when the biased field is parallel to the direction of applied stress, for a positive magnetostrictive material, the increasing tensile force will increase the signal amplitude of magnetic absorption. On the contrary, the increasing compressive force will reduce the signal amplitude of magnetic absorption; for a negative magnetostrictive material, the the increasing tensile force will reduce the signal amplitude of magnetic absorption. Contrarily, the increasing compressive force will increase the signal amplitude of magnetic absorption. When the biased field is perpendicular to the direction of applied stress, the influence of stress on the peak-peak signal amplitude will be just contrary to the above situations. As a matter of fact, the stress direction of ferromagnetic material can be quantitatively measured by measuring the magnetic absorption signal, but it is not easy to realize the realtime stress testing for a ferromagnetic member.

\section{Conclusions}

In regard to the magnetic nondestructive testing on damages and stresses of ferromagnetic member, nowadays some researchers and experts have carried out lots of researches, obtained an exciting achievements, but most of their researches are rather accentuated on aviation, machinery, petrol etc. and are mainly corresponding to the magnetic nondestructive qualitative testing of damages and stress for ferromagnetic member. However, in the field of Civil Engineering, regarding the magnetic nondestructive testing on damages and internal forces distributions for ordinary constructional steel, the present research is almost seldom seen, especially for the researches on realtime stress magnetic nondestructive testing for steel structure and steel members, the involved documents are even fewer. Therefore, the unique magnetomechanical effect of constructional steel, the relationship between the stress and its magnetic properties, particularly the relationship of the stress versus the magnetic flux change, and how to realize a realtime stress magnetic nondestructive testing for steel structures or steel members are equally worth further exploring.

\section{Acknowledgements}

The author would like to acknowledge the financial support from the National Natural Science Foundation of China (Nos. 51108035, 51208058, 51178388 and 10972168), the China Postdoctoral Science Foundation funded project (No. 20100481313, 2011049165 and 2012T50790), the Natural Science Basic Research Plan in Shaanxi Province of China (No. 2010JQ7006), and the Special Fund for Basic Scientific Research of Central College (No. CHD2012ZD012).

\section{References}

1. J. Anglada-Rivera, L.R. Padovese, J. Capó-Sánchez, J. MAGN. MAGN. MATER. 231, 299 (2001)

2. V. Babbar, B. Shiari, L. Clapham. IEEE Transaction on Magnetics 40:1, 43-49. (2004)

3. A. Dhar, L. Clapham, D.L. Atherton, NDT \& E Int. 34, 507 (2001)

4. L.H. Dong, B.S. Xu, S.Y. Dong Nondestructive Testing 28, 245 (2006) (in Chinese)

5. J. Du, Master Thesis, Huazhong University of Science and Technology (2004) (in Chinese)

6. A.A. Dubov, Weld. World 46, 317 (2002)

7. A.A. Dubov, Tyazheloe Mashinostroenie 6, 13 (2005)

8. A.A. Dubov, Met. Sci. Heat Treat + 39, 401 (1997)

9. A.A. Dubov, A Method of Definition of Stressed-Strained State of Product by Scattering Magnetic Fields: Russia, Patent 2000119881/28(020902) (2000)

10. A.A. Dubov, Therm. Eng. 48, 289 (2001)

11. A.A. Dubov, V.G. Kouleev, $2^{\text {nd }}$ International Conference on Diagnostics of the Equipment and Constructions with Usage of Metal Magnetic Memory, Moscow, Russia, 32 (2001)

12. A.A. Dubov, E.A. Demin, $1^{\text {st }}$ Internatioal Conference on Diagnostics of the Equipment and Constructions with Usage of Metal Magnetic Memory, Moscow, Russia, 24 (1999)

13. A.A. Dubov, G.V. Vstovsky, Inspection Diagnostics 3, 247 (1999)

14. A.A. Dubov, A.lA. Dubov, S.M. Kolokolnikov, Method of Metal Magnetic Memory (MMM) and Inspection Devices. Training handbook (Moscow: Tisso Co., 2003)

15. F. Foster, Defecktopia 11, 3 (1982)

16. A. Gilanyi, K. Morishita, T. Sukegawa, Fusion Eng. Des. 42, 485 (1998)

17. S.P. Gwan, S.P. Eun, IEEE Trans. Magn. 38, 1277 
(2002)

18. B.L. Hou, J.P. Zhou, X. PENG, Journal of Experimental Mechanics 13, 98 (1998) (in Chinese)

19. C. Jagadish, L. Clapham, D.L. Atherton, IEEE Trans. Magn. 26, 1160 (1990)

20. D.C. Jiles, IEEE Trans. Magn. 29, 343 (1996)

21. J.H. Jin, Y.H. Kang, S.Z. Yang, Chinese Journal of Scientific Instrument 22, 469 (2001) (in Chinese)

22. G.M.W. Jonathan, Spatial Structure 11, 63 (2005) (in Chinese)

23. J.B. Ju, J.S. Lee, J.I. Jang, Int. J. Pres. Ves. Pip. 80, 641 (2003)

24. T.W. Krause, L. Clapham, A. Pattantyus, J. Appl. Phys. 79, 4242 (1996)

25. J.W. Li, J.M. Chen. Handbook of Nondestructive Testing (Beijing: China Machine Press, 2002) (in Chinese)

26. L.M. Li, S.L. Huang, Nondestructive Testing 25, 387 (2003) (in Chinese)

27. S.P. Li, Master Thesis, Tianjin University, (2004) (in Chinese)

28. M. Lindgren, T. Lepisto, NDT \& E Int. 34, 337 (2001)

29. H. Liu, Master Thesis, Hefei University of Technology (2002) (in Chinese)

30. X.Y. Ma, D.Q. Sun, B.W. Qiu, Journal of Huazhong University of Science and Technology 24, 40 (1996) (in Chinese)

31. G.A. Maugin, M. Sabir, Int. J. Plasticity 6, 573(1990)

32. X.R. Mu, Nondestructive Testing 11, 229 (1989) (in Chinese)

33. D.G., C.G. Park Kim, J.H. Hong, J. Magn. Magn. Mater. 215, 765 (2000)
34. J. Philip, C.B. Rao, T. Jayakumar, NDT Int. 33, 289 (2000)

35. A. Plotnikov, L. Clapham, Insight 44, 74 (2002)

36. J.L. Ren, J.M. Lin, Metal Magnetic Memory Testing Technique (Beijing: China Electric Power Press, 2002) (in Chinese)

37. D.M. Stewart, K.J. Stevens, A.B. Kaiser, Curr. Appl. Phys. 4, 308 (2004)

38. V. Thomas, G. Silvio, the $9^{\text {th }}$ European Conference on NDT, Berlin, Germany, 1 (2006)

39. C.L. Wang, S.Y. Liang, X.Z. Zuo, Journal of Ordnance Engineering College 19, 13 (2007) (in Chinese)

40. W. Wang, S.Q. Su, S.L. Wang, Journal of Xi'an University of Architecture and Technology 37, 322 (2005) (in Chinese)

41. Y.D. Wang, Master Thesis, Daqing Petroleum Institute (2004) (in Chinese)

42. L.R. Willam, Magabsorption NDE in Metals Handbook (9th Edition. Vol. 17, NDE and Quality Control, American Society for Metals, 1989)

43. Y. Xie, C.N. Peng, Journal of Jimei University 6, 322 (2001) (in Chinese)

44. H.Y. Xing, M.Q. Xu, R.X. Wang, Key Eng. Mater. 324, 619 (2006)

45. W.M. Zhang, X. Guo, J.J. Yuan, Nondestructive Testing, 28, 623 (2006) (in Chinese)

46. X.Q. Zhu, Journal of Experimental Mechanics 6, 327(1991) (in Chinese). 